\title{
NOISES OF ELECTRONIC DEVICES (GENERAL INFORMATION, SOURCES)
}

\author{
Gulkhayo Oybekovna Akbarova \\ Teacher of Andizhan State University, Republic of Uzbekistan
}

Article DOI: https://doi.org/10.36713/epra4857

\begin{abstract}
In the article have been shown the analysis of the main approaches to the classification of internal electrical noise in electronic semiconductor devices. Classifications of electrical noise for each of the selected features are presented. KEY WORDS: Thermal noise, flicker noise, Gaussian noise, white noise, frequency noise.
\end{abstract}

ABSTRACT

\section{INTRODUCTION}

The physical processes that were considered in the study of electronic devices were considered deterministic, although in fact these processes are influenced by various random factors causing fluctuations (random changes) in the currents and voltages in the circuits of the devices. Fluctuations in voltage (or current) at the terminals of electronic devices and devices are usually called noise. Noises are inherent in all electronic devices, and they are caused by the random nature of the movement (at the microscopic level) of charge carriers inside electronic devices. Noise is often a factor imposing restrictions on the characteristics of electronic devices, in particular, on their sensitivity. Sometimes noise can be used as a means to study the electrical characteristics of the system itself.

\section{METHODS}

Usually noises in electronic devices are considered as stationary random processes in the time or frequency domain, so the main characteristics of noise are: the average value of the implementation of this random process (expectation); spectral density giving the average spectral component of the fluctuating signal; variance characterizing the energy of noise with a mathematical expectation (Average value) equal to zero; an autocorrelation function that determines the statistical relationship between two values of a random function $x(t)$, separated by a time interval $T$, and a number of other characteristics, the meaning and value of which will be given as they are used.

\section{RESULTS AND DISCUSSIONS}

The main types of noise that appear in electronic devices include: thermal, diffusion, shot, generation recombination, $1 / f$ noise, current distribution noise, noise of secondary emission, avalanche multiplication, explosive, ionic and some others [1].

Thermal noise arises due to random fluctuations in the velocity of charge carriers in the medium in the device. Carrier velocity fluctuations, in turn, cause random changes in the carrier density and, as a consequence of these processes, current and voltage fluctuations appear. Thermal fluctuations do not violate the thermal equilibrium of the system, since a random (microscopic) deviation of an electro physical parameter from the equilibrium state is followed by an (average) return to it. A large number of microscopic events of this kind lead to a noticeable change in the current or voltage at the terminals of the device, resistor, etc. 
Figure 1.

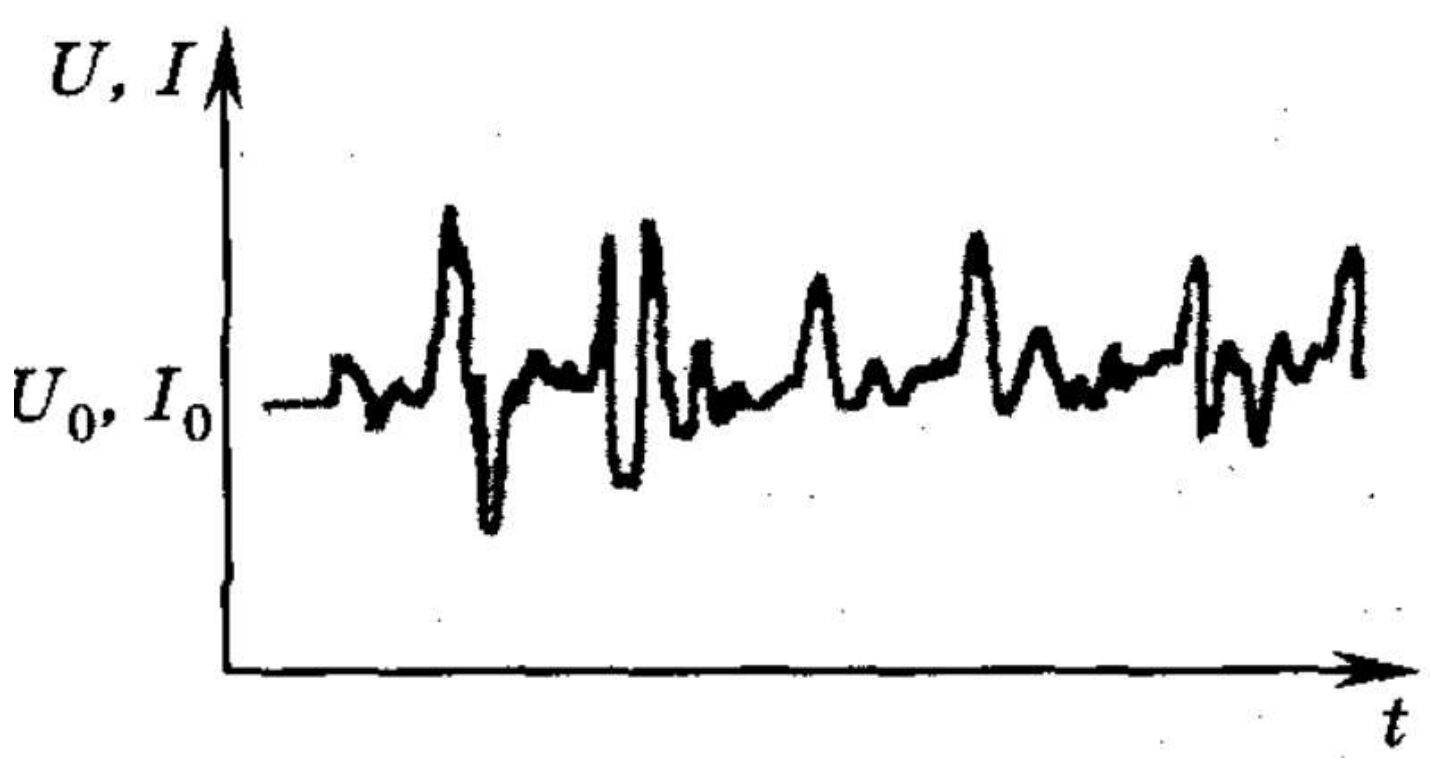

Shot noise is associated with the passage of free charge carriers through a potential barrier. It was first discovered in electron tubes. The name of this type of noise is due to the fact that the sound from the speaker installed at the output of the tube amplifier, at a high gain in the absence of a useful signal at the input, resembled the sound of a fraction falling on a steel sheet. Simple shot noise represents current fluctuations caused by electrons or holes, which are emitted randomly and independently from each other, without interacting with each other. In solid state devices, for example, diodes and transistors, carriers from the emitter exit randomly and move through the potential barrier of the depleted $p-n$ junction layer, causing shot noise. In vacuum and gas-discharge devices, electrons are randomly emitted from the cathode and then moved to the anode under the influence of an electric field. These electrons randomly overcome the potential barrier formed at the boundary of the cathode with a vacuum or a working medium. Both in solid-state and in vacuum devices, the current generated by carrier flows fluctuates around an average level, which is associated with the discrete nature of emission (see Fig. 1). Although the physical nature of thermal and shot noise is different, the form of noise signals is similar in both cases [2]. In either case, it can be represented as a large sequence of random impulses that are similar in shape and randomly distributed over time.

However, we emphasize that shot noise in lamps, in contrast to thermal noise in conductors, occurs during a substantially no equilibrium process, in which the movement of carriers is due to an external field and there is completely no interaction of carriers with both the medium and each other.

Diffusion noise is due to the probabilistic nature of charge carrier diffusion. For semiconductor materials, this type of noise is the cause of thermal noise, and for devices with $\mathrm{p}-\mathrm{n}$ junctions, it is the main component of shot noise.

Current noise, or $1 / f$ noise, flicker noise, contact noise, excess noise - these are all different names of $1 / f$ noise [3]. This name is due to the fact that the spectral power density of this noise varies depending on the frequency according to the law $f-\alpha$, where $\alpha \sim 0.8 \ldots 1.2$.

From the side of ultra-low frequencies, this dependence is observed up to $f \sim 10-6 \mathrm{~Hz}$. At high frequencies, $1 / f$ noise is masked by thermal, shot or some other type of noise. At the moment, there is no unified theory of this phenomenon, although individual particular models can describe such noise quite well in some types of devices. Apparently, $1 / f$ noise is a combination of a sufficiently large number of different physical phenomena that obey the same regularity.

Based on the available data, it can be concluded that the causes of $1 / f$ noise in different cases are different. $1 / f$ noise can be represented as a random sequence of pulses of a certain shape $u(t) \sim$ $t-1 / 2 U(t)$ where $U(\mathrm{t})$ is the unit step function: $U(t)$ $=1$, for $1>0, U(t)=0$ for $t<0$, for which the spectral density varies as $f-1$ in a wide frequency range. Such a formal representation does not yet provide an idea of the physical mechanism that generates impulses of this form. Noise obeying the law when the spectral density is inversely proportional to the frequency appears in all materials 


\section{SJIF Impact Factor: 7.001| ISI I.F.Value:1.241| Journal DOI: 10.36713/epra2016 \\ ISSN: 2455-7838(Online) \\ EPRA International Journal of Research and Development (IJRD)}

Volume: 5 | Issue: 7 | July 2020

- Peer Reviewed Journal

and elements used in electronics: intrinsic semiconductors, resistors, devices on $p-n$ junctions, films, liquid metals and solutions of electrolytes, lamps with thermal cathodes, superconductors and Josephson transitions, etc. Despite a very large number of experimental facts, it is still not possible to say with certainty whether $1 / f$ noise belongs to volumetric or surface phenomena. In some types of devices, for example, MIS transistors, this is surface Effect. In this case, the semiconductor - oxide interface plays an important role in the occurrence of noise. For other types of devices, for example, for homogeneous resistors, this is a volumetric effect due to fluctuations in the number or mobility of charge carriers. Sometimes the obtained experimental data speak in favor of fluctuations of both the number and mobility of carriers at the same time. The most reliable data on the spectral density of $1 / f$ noise in homogeneous materials. There is an empirical law according to which the spectral density of $1 / f$ noise is inversely proportional to the total number of charge carriers in the sample. Although there is no theoretical justification for this law and there is no connection with any physical mechanism for the occurrence of $1 / f$ noise, it apparently reflects a characteristic feature of many phenomena accompanying $1 / f$ noise. In substantiating this empirical law, the most widely discussed mechanism was the presence of traps on the surface and the exchange of energy of the device or material with the environment during thermal equilibrium.

As already noted, the physical mechanism responsible for $1 / f$ noise can manifest itself in each case in the characteristic features of the shape of the noise signal; otherwise, it is assumed that the analysis of the mathematical description of a process with the characteristics of $1 / f$ noise can lead to an understanding of physics underlying this phenomenon. Currently, two process models having the shape of a $1 / f$ spectrum are most widely used:

1) A model of a random train of pulses;

2) A model based on a superposition of a large number of relaxation processes with a wide variation of characteristic time constants.

The second model is used more widely, since it is directly related to $1 / f$ noise in MOS transistors associated with surface phenomena. In accordance with this mechanism, carriers tunnel between the energy states of the semiconductor and the levels of traps localized in the dielectric (oxide) layer, which is confirmed by the practical absence of $1 / f$ noise in field effect transistors (PTs) with pn junctions, where surface phenomena are weakly manifested. At the same time, in a MOSFET with a large semiconductor - oxide interface, the component caused by this type of noise is dominant in the overall noise spectrum. As the experiment shows, in this case, the noise intensity is extremely sensitive to the quality of the surface, and the spectral dependence, as a rule, remains the same, i.e., $\sim 1 / f$, regardless of the state of the surface. Therefore, each new, subsequent oxide layer causes the appearance of an intrinsic $1 / f$ spectrum, and does not shift the HF boundary of the already existing spectrum towards lower frequencies due to the large time constants of deeper traps. The widely accepted McWarter theoretical model, based on carrier trapping by surface traps, describes only a very particular noise mechanism in semiconductors. The presence of $1 / f$ noise in metals and other materials, the strong temperature dependence of $1 / f$ noise in metal films, and a number of other processes with a $1 / f$ spectral dependence have not yet been completely explained [4].

Generation-recombination noise arises in the process of generation and recombination of both equilibrium and no equilibrium carriers. In these processes, electrons and holes appear and disappear randomly. The random nature of the appearance and disappearance of free carriers leads to fluctuations in the resistance of the semiconductor or in individual areas of the device, where the processes of recombination and generation of free carriers are realized. If a direct current is passed through a sample of such a semiconductor, then at its ends, in addition to the constant, the fluctuation component of the also arises.

Most semiconductor devices have carrierdepleted regions, such as electrical transitions of various kinds. When carriers diffuse from one or another bulk region into the depletion layer, carriers can cross and leave it, or be reflected from it, or, finally, some of the carriers can be captured by recombination centers (traps) inside the layer under consideration. In the latter case, current pulses will appear in the external circuit, which form a recombination current (see $\S 1.3,2.5$ ). This current consists of a stationary component, on which fluctuations are superimposed, due to the random nature of the recombination. If the carriers are formed in the depleted layer, then the electric field in it separates the carriers and makes them move in the direction of those bulk regions where they are the main ones.

The generation processes are accompanied by the appearance of current pulses in the external circuit that have the opposite sign compared to pulses arising from recombination. The generation current also consists of a stationary component and superimposed generational fluctuations distributed according to a random law. Thus, the process of generation and recombination of carriers is associated with their random appearance and disappearance, and these phenomena can be considered as a sequence of independent random events similar to shot noise. 


\section{SJIF Impact Factor: 7.001| ISI I.F.Value:1.241| Journal DOI: 10.36713/epra2016 \\ ISSN: 2455-7838(Online) \\ EPRA International Journal of Research and Development (IJRD) \\ Volume: 5 | Issue: 7 | July 2020}

- Peer Reviewed Journal

Therefore, to describe generation-recombination noise, one can often use formulas obtained for the analysis of shot noise with the introduction of appropriate corrections that take into account the specifics of the process.

Explosive noise is manifested, as a rule, in devices such as diodes, transistors at $p-n$ junctions, tunneling diodes, and composite resistors. In its simplest form, explosive noise appears as a bi stable signal (with two levels) of a stepped shape, with small changes in amplitude and with randomly distributed time intervals between steps (like a random telegraph signal). However, sometimes there are signals with several levels of amplitudes (steps). It is assumed that the explosive noise at reverse biased $p-n$ junctions is due to the irregular switching on and off of the surface conduction channels. With direct switching on, the cause of this noise is crystal defects in the transition region, and the main role is played not by metallic impurities, but by defects like dislocations.

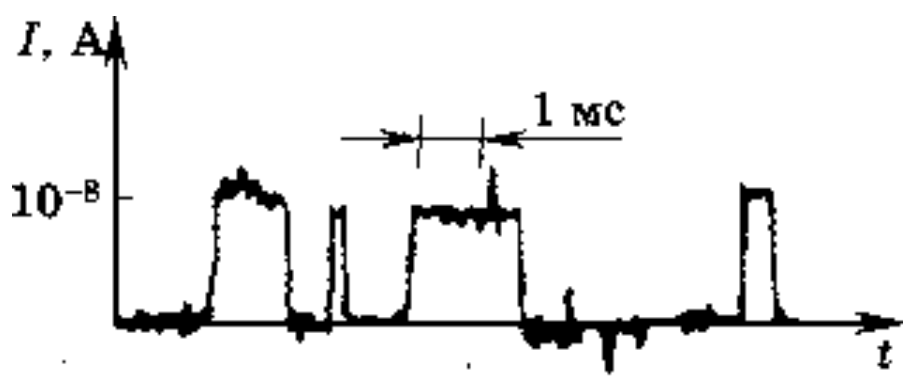

Figure 2

A typical implementation of bi stable explosive noise is shown in Fig. 2. It consists of random step emissions with white noise (having a uniform spectrum in a wide frequency range from $O$ to $1013 \mathrm{~Hz}$ ). It is believed that the signal is symmetric if the average duration time of each of the two levels of the stage is the same, and asymmetric if there is a significant deviation from such a condition.

Avalanche and micro plasma noise are associated with avalanche multiplication, which takes place in regions of a strong electric field, where the kinetic energy of free charge carriers is sufficient for ionization of the atoms of the crystal lattice. The ionization processes underlying the multiplication of carriers occur randomly, thus creating noise in the current of avalanche ionization. The avalanche multiplication of carriers finally leads to a breakdown of the $p-n$ junction. The generation of electron-hole pairs during multiplication ultimately forms an electron-hole plasma. Quite often, breakdown processes are not realized over the entire transition area, but in separate, sometimes microscopically small areas, where, due to various kinds of defects, the greatest electric field strength exists and micro plasma is formed as a result of breakdown. The realization of micro plasma noise is often similar to the realization of explosive noise, but with an amplitude much larger $(\sim 10-5 \mathrm{~A})$, while the amplitude of the explosive noise signal is $10 \sim 8 \mathrm{~A}$ in order of magnitude.

The micro plasma itself is localized inside the transition in regions of a strong electric field with characteristic dimensions of several hundred angstroms, in which cracks and other defects of the crystal lattice contain traps. These traps, capturing free carriers, form an increased charge density, which causes the appearance of a strong field. The formation and subsequent destruction of micro plasma is a random process, which leads to the observed stepwise changes in the transition current.

Current distribution noise occurs when current is shared between the electrodes of a device, such as a transistor or pentode. The nature of the current distribution is random in nature, mainly due to fluctuations in the transverse component of the electron velocity. These noises are also influenced by fluctuations in the cathode current or emitter current, potential fluctuations on the electrodes, various field in homogeneities, instabilities of the electro physical parameters of the electrodes, etc.

All these reasons lead to a random change in the direction of motion of the electrons and, as a consequence, the interception of electrons by a grid in an electric vacuum tube or other electrode in another type of device is random, which causes current fluctuations in the electrode circuits.

Secondary emission noise is due to the fact that the secondary emission coefficient from the electrodes of electron tubes is variable over time due to the instability of the primary electron current, surface imperfections, the presence of various impurities, in homogeneities, and foreign inclusions in the material of the secondary cathode. In conventional receive-amplification lamps, noise caused by secondary emission is usually much less than shot noise and current distribution noise.

Radiation (photon) noise is determined by fluctuations in the parameters of the light signal 
(radiation) incident on the receiver, i.e., fluctuations in the number of photons incident on the photosensitive layer of the photo detector that come from the emitter and elements of the receiver itself. In addition to those considered, in electronic devices there are some other types and sources of noise.

\section{CONCLUSION}

The analysis showed that in the scientific literature there are four main approaches to the classification of electrical noise in semiconductors:

1) Classification by the physical nature and mechanism of occurrence;

2) Classification according to the behavior of spectral density and probability density;

3) Classification from the point of view of the theory of random processes;

4) Classification by external manifestation.

In cases where the subject of the study is the noise itself and the causes of their occurrence, classifications are used according to the first three signs. In cases where the subject of the study is to reduce the noise level in electronic devices, use the classification of noise by external manifestation.

\section{REFERENCES}

1. Van der Zil A. Noise. Sources, description, measurement. M.: Sov. radio, 1973.

2. Van der Zil A. Fluctuations in radio engineering and physics. M.; L.: Gosenergoizdat, 1958.

3. Horowitz P., Hill W. The Art of Circuitry. Moscow: World, 2003.

4. G.G. Shishkin., A.G. Shishkin. Electronics. Moscow: Bustard, 2009. 